EMMA CROSBIE (Orcid ID : 0000-0003-0284-8630)

DR. JAMES MICHAEL NIALL DUFFY (Orcid ID : 0000-0001-6127-9859)

Article type : Systematic review

\title{
Research priority setting in women's health: A systematic review
}

Lois Graham ${ }^{1}$, Benjamin J. G. Illingworth ${ }^{2}$, Marian Showell ${ }^{3}$, Melissa Vercoe ${ }^{3}$, Emma J. Crosbie

4 , Lynda J. Gingel ${ }^{5}$, Cynthia M. Farquhar ${ }^{3}$, Andrew W. Horne ${ }^{6}$, Mathew Prior ${ }^{7}$, Judith M.

Stephenson ${ }^{8}$, Laura A. Magee ${ }^{9}$, James M. N. Duffy 8,10

${ }^{1}$ Christ Church, Oxford University, Oxford, United Kingdom.

2 North West Anglia NHS Foundation Trust, Peterborough City Hospital, Peterborough, United Kingdom.

${ }^{3}$ Cochrane Gynaecology and Fertility Group, University of Auckland, Auckland, New Zealand.

${ }^{4}$ Department of Obstetrics and Gynaecology, Manchester University NHS Foundation Trust, Manchester Academic Health Sciences Centre, Manchester, United Kingdom.

${ }^{5}$ Radcliffe Women's Health Patient and Public Participation Panel, University of Oxford, Oxford, United Kingdom.

${ }^{6}$ MRC Centre for Reproductive Health, University of Edinburgh, Edinburgh, United Kingdom.

${ }^{7}$ Newcastle Fertility Centre, Newcastle Upon Tyne Hospitals NHS Foundation Trust, Newcastle, United Kingdom.

8 Institute for Women's Health, University College London, London, United Kingdom.

9 Department of Women and Children's Health, School of Life Course Sciences, King's College London, London, United Kingdom.

${ }^{10}$ Barking, Havering and Redbridge University Hospitals NHS Trust, Romford, Essex, United Kingdom.

This article has been accepted for publication and undergone full peer review but has not been through the copyediting, typesetting, pagination and proofreading process, which may lead to differences between this version and the Version of Record. Please cite this article as doi: $\underline{10.1111 / 1471-0528.16150}$

This article is protected by copyright. All rights reserved 


\section{Correspondence to}

Dr James M. N. Duffy DPhil MRes PG HCL MBChB BSc (Hons) Institute for Women's Health, University College London London WC1E 6BT United Kingdom +447949066806 james.duffy3@nhs.net @jamesmnduffy

\section{Running title}

Research priority setting in women's health

\section{Abstract}

Background: Developing a shared agenda is an important step in ensuring future research has the necessary relevance.

Objective: To characterise research priority setting partnerships (PSP) relevant to women's health.

Search strategy: Included studies were identified by searching MEDLINE and the James Lind Alliance (JLA) database.

Selection criteria: PSP which used formal consensus methods.

Data collection and analysis: Descriptive narrative to describe the study characteristics, methods, and results.

Results: Ten national and two international PSPs were identified. All PSPs used the JLA method to identify research priorities. Nine PSPs had published a protocol. Potential research uncertainties were gathered from guidelines (two studies), Cochrane reviews (five studies), and surveys (12 studies). The number of healthcare professionals (range 31 to 287), patients (range 44 to 932 ), and others (range 33 to 139) who responded to the survey, and the number of uncertainties submitted (range 52 to 4,767 ) varied. All PSPs entered confirmed research uncertainties (range 52 to 4,767 ) into interim priority setting surveys and healthcare professionals (range 31 to 287), patients (range 44 to 932), and others (range 33 to 139) responded. All PSPs entered a short list of research uncertainties into a consensus development meeting, which enabled healthcare professionals (range six to 21), patients (range eight to 14), and others (range two to 13) to identify research priorities (range 10 to 15). Four PSPs have published their results. 
Conclusions: Future research priority setting studies should publish a protocol, use formal consensus development methods, and ensure their methods and results are comprehensively reported.

Funding: Catalyst Fund, Royal Society of New Zealand.

Keywords: Consensus methods, James Lind Alliance, Nominal Group Technique, priority setting partnerships, and research priorities.

Tweetable abstract: Research published in @BJOGtweets highlights future research priorities across women's health including @FertilityTop10

\section{Introduction}

The ultimate aim of clinical research is to improve care and patient outcomes. For this to be possible, research should address questions that are pertinent to healthcare professionals, 
women, and clinical practice, answer these questions using the most appropriate scientific methods, and report research results in a comprehensive, transparent, and accessible manner. ${ }^{1}$ Without engaging in these steps there is potential for significant research waste. ${ }^{2}$ The first step in the research production cycle is to identify an appropriate question. Before the recent emphasis on patient and public involvement, research funding organisations and researchers identified, refined, and prioritised their own research agenda. As a result, there has been a stark contrast between what research is needed and what research has been initiated, conducted, and reported. ${ }^{2}$ For example in the case of osteoarthritis Tallon and colleagues have demonstrated a mismatch between the agendas of the research community, healthcare professionals, and people with the disease. ${ }^{3}$ They concluded if this apparent supply and demand gap was not addressed, then embedding evidence-based medicine within routine clinical practice would be frustrated. ${ }^{3}$

Over the last decade, Sir lan Chalmers, founder of the Cochrane Collaboration, has advocated for research priorities to be jointly identified by healthcare professionals, patients, and communities. ${ }^{4}$ Sir lan established the James Lind Alliance which brings healthcare professionals, patients, and communities together, in priority setting partnerships. ${ }^{4}$ Each partnership identifies future research priorities in a particular area of health and social care. Using formal consensus methods advocated by the James Lind Alliance, priority setting partnerships engage in an open and transparent process to identify and prioritise unanswered research questions, known as research uncertainties (Figure 1). ${ }^{5}$ The expectation is that prioritised research uncertainties will establish the future research agenda of funding organisations and researchers. As a result, it is hoped that the gap will close between what research is needed and what research is produced.

\section{Methods}

A protocol with explicitly defined objectives, criteria for selection, and approaches to data extraction was developed. The objectives of this systematic review fell outside the scope of the International Prospective Register of Systematic Reviews (PROSPERO) as they invite researchers to register reviews which aim to influence a health related outcome and therefore did not require registration. ${ }^{6}$

A systematic literature review was undertaken by searching MEDLINE for relevant studies from inception to June 2019 (Appendix S1), as well as the James Lind Alliance database (www.jla.nihr.ac.uk/priority-setting-partnerships). This James Lind Alliance database is an inventory of research priority setting studies. ${ }^{5}$ Research priority setting studies relevant to women's health that engaged with formal consensus development methods and included 
healthcare professionals and women were included. No language or publication date restrictions were applied. Two reviewers (BI and IG) independently screened titles and abstracts. They critically reviewed the full text of selected studies to assess eligibility. Any discrepancies between the reviewers were resolved by discussion.

Using a pilot-tested and standardised data extraction form, two researchers ( $\mathrm{BI}$ and IG) independently extracted study characteristics, methods, and results. For published research priority setting studies, characteristics, quantitative, and qualitative methods identifying potential research uncertainties, and consensus methods to prioritise unanswered research questions, known as research uncertainties, were extracted.

This study was reported in accordance with the Preferred Reporting Items for Systematic Reviews and Meta-Analyses (PRISMA) statement. ${ }^{7}$ Descriptive narratives were reported to characterise research priority setting studies, mapping their characteristics, methods, and results.

LG, patient and public advisor, contributed to prioritising the research question, interpreting the data, and provided critical revision of the manuscript for intellectual content.

This study was supported by the Catalyst Fund, Royal Society of New Zealand, awarded following external peer review. The funder had no role in the study design, data collection and analysis, decision to publish, or preparation of the manuscript.

\section{Results}

The Medline search identified 4,066 records that were screened by two authors independently (Figure S1). Thirty-seven full text articles were assessed for eligibility. Seven research priority setting studies were identified. ${ }^{8-14}$ An additional four research priority setting studies were identified in the James Lind Alliance database. ${ }^{15-18} \mathrm{~A}$ further research priority setting study was identified through personal communication with the clinical lead. ${ }^{19}, 20$ Eight completed and four progressing research priority setting studies were included (Table 1 and Table S1). ${ }^{8-19}$ The priority setting partnership for infertility has established the top ten research uncertainties for male infertility, female and unexplained infertility, medically assisted reproduction, and ethics, access, and organisation of care in a single initiative. ${ }^{21}$ Four research priority setting studies have reported their full results in a peer reviewed publication. ${ }^{10,11,13,14}$

This article is protected by copyright. All rights reserved 
Three research priority setting studies were relevant to benign gynaecology and infertility, establishing research priorities for miscarriage ${ }^{14}$, infertility ${ }^{21}$, and endometriosis ${ }^{8}$. Seven research priority setting studies were related to pregnancy and childbirth, including studies establishing research priorities for gestational diabetes ${ }^{11}$, preterm birth ${ }^{12}$, and stillbirth ${ }^{13}$. A single research priority setting study, establishing research priorities for endometrial cancer, was related to gynaecological oncology. ${ }^{14}$ Sexual and reproductive health was under-represented, with a single research priority setting study related to contraception being identified. ${ }^{15}$ Nine research priority setting studies were conducted in the United Kingdom. 8 , 10, 12-18

All studies used the James Lind Alliance method (Figure 1). ${ }^{11}$ Nine research priority setting studies followed a protocol. ${ }^{8-10,13,14,16-18}$ When considering completed research priority setting studies, there was considerable variation in the number and range of stakeholders who participated in gathering potential research uncertainties (Table S2), interim priority (Table S3), and final priority setting.

Research priority setting studies gathered research uncertainties from clinical practice guidelines $^{10,21}$ and Cochrane systematic reviews ${ }^{10,11,13,14,21}$. All priority setting studies gathered potential research uncertainties through electronic surveys. There was considerable variation in the number of: (1) respondents who completed the surveys (range 391 to 1208 ); (2) responses from individual stakeholder groups, including healthcare professionals (range 31 to 287), patients (range 44 to 932), and others (range 33 to 139); and (3) submitted research questions (range 52 to 4 767). Three research priority setting studies published the long list of collated research uncertainties. ${ }^{10,13,14}$

All research priority setting studies used an interim prioritisation survey to reduce the long list of research uncertainties to a short list. The number of research uncertainties entered into the interim prioritisation survey ranged from 39 to 104 . There was substantial variation in the number of respondents who completed the surveys (range 8 to 2 103) and the responses from individual stakeholder groups, including healthcare professionals (range 3 to 749), patients (range 3 to 1 797), and others (range 2 to 135). There was variation in the methods used to rank the long list of research uncertainties across studies (Table S3).

Between 15 and 29 research uncertainties were entered into face-to-face consensus development meeting. Using the Nominal Group Technique, healthcare professionals, patients, and others further prioritised the research uncertainties. The number of participants in each 
stakeholder group varied across research priority setting studies (Table S3). By the end of the consensus development meeting research uncertainties were prioritised, for example ten research uncertainties were prioritised for female and unexplained infertility ${ }^{21}$ (Figure 2), miscarriage ${ }^{10}$, and endometriosis ${ }^{8}$.

The priority setting partnership for contraception has extended their original work by publishing an explanatory note for each prioritised research uncertainty which provides contextual information, a review of current research evidence including justification of uncertainty, and narrative from the initial survey gathering research uncertainties from healthcare professionals and people with lived experience of contraception. ${ }^{22}$ The priority setting partnership for miscarriage has published similar information. ${ }^{23}$

\section{Discussion}

\section{Main findings}

Eight research priority setting studies have been completed and research priorities for twins and multiple pregnancies, hyperemesis gravidarum, blood pressure in pregnancy, and diabetes in pregnancy are being developed. Although research priorities have been developed in a range of different health conditions relevant to women's health, several areas are currently under-represented, including benign gynaecology, urogynaecology, sexual and reproductive health, gynaecological oncology, and LGBTQ+ health. All research priority setting studies have used the James Lind Alliance method. When considering completed research priority setting studies, there was considerable variation in the number and range of stakeholders who participated in gathering potential research uncertainties, interim priority, and final priority setting. Two research priority setting studies have published an explanatory note for each prioritised research uncertainty providing contextual information pertaining to the level of uncertainty the research priority is hoping to address.

\section{Strengths and limitations}

To our knowledge, this is the first systematic review to characterise and evaluate ongoing and published research priority setting studies relevant to women's health. The strengths of this review include its comprehensive search strategy and design ensuring the review process and data extraction were conducted independently by two authors. 
This empirical evaluation is not without limitations. The search strategy was limited to the bibliographical database MEDLINE and James Lind Alliance database. Additional research priority setting studies could have been identified by expanding the search to other bibliographical databases. However, identifying research priority setting studies is challenging, as no appropriate medical subject heading exists, and prospective registration is not compulsory.

The majority of completed research priority setting studies had not published a comprehensive report and accurately assessing their methods and results was challenging. Future priority setting studies should be required to publish their full study report within a peer reviewed journal. There are currently no established criteria to assess the quality of completed research priority setting studies. No decisions regarding the quality of research priority setting studies and subsequent research priorities can confidently be made. It is unclear to what extent the variation in the methods used to gather research uncertainties, the consensus methods used to priorities research uncertainties, and the use of a trained James Lind Alliance advisor, would affect the final research uncertainties prioritised. Further research is needed to develop an objective tool to assess the methodological quality of these studies.

\section{Interpretation}

Although prospective registration is not currently mandated for research priority setting studies, its implementation could help prevent unnecessary duplication of research effort, assist key stakeholders to identify planned or ongoing research priority setting studies, and ensure the design of new research studies is informed by relevant research priorities. Publishing research priority setting study protocols could provide an additional strategy to improve research priority setting research in a similar fashion as prospective registration. Researchers would be able to obtain feedback on draft protocols through peer review and enable consumers to compare what was originally intended with what was actually. Future research priority setting studies should be strongly encouraged to publish their results within a peer reviewed journal. The published report should include information justifying the selection and ranking of the prioritised research uncertainties and commentary pertaining to discussion surrounding the research uncertainties which were not prioritised.

The James Lind Alliance has published guidance to inform the design of research priority setting studies. However, there is considerable uncertainty regarding the number and characteristics of participants required, the optimal consensus development methods, and the most efficient approaches to establish a prioritised list of research uncertainties. This reflects the limited 
methodological research which has been undertaken to understand the most appropriate methods to develop research priorities. When considering the initial survey gathering potential research uncertainties, the James Lind Alliance guidebook states that this initial survey should be considered a qualitative research study aiming to engage a range of possible stakeholders from diverse geographical locations. ${ }^{5}$ As in all types of qualitative research studies, priority setting partnerships should consider aiming for quality not quantity and use appropriate methods to demonstrate data saturation. ${ }^{24}$ When considering the prioritisation survey, the James Lind Alliance guidebook states that a formal consensus method, the modified Nominal Group Technique method, should be used. ${ }^{5}$ The modified nominal group technique does not depend on statistical power. Working from its underlying principles, group error should reduce and the decision quality increase as the number of participants increases. There is no robust method for calculating the required number of participants. Between ten and 15 participants has yielded sufficient results and assured validity in other settings. ${ }^{25}$

Future priority setting partnerships should ensure access to high quality advice regarding formal consensus methods. Possible directions for future methodological research could include evaluating different methods to recruit survey respondents, for example, using automated bots to allow potential survey respondents to answer questions regarding the study, assessment of the feasibility of identifying potential research uncertainties from published randomised trial reports, and comparing virtual and face-to-face meetings in developing consensus research priorities. Evaluating different approaches to priority setting, including the modified Delphi method, modified Nominal Group Technique, or consensus development workshop, would also be informative when designing future priority setting studies. ${ }^{25}$

In the last five years, the number of research priority setting studies has risen. There is evidence research funders and individual researchers have sought to address prioritised research uncertainties (Figure 2). Further research priority setting studies are required across women's health, including fibroids, menopause, and gynaecological cancer. The development of infrastructure to evaluate, disseminate, and implement research priorities within women's health is required to ensure effective and timely discussions which could inform the prioritisation of future research. Only two research priority setting studies have published contextual information pertaining to the level of uncertainty the research priority is hoping to address. Future studies should be encouraged to provide such information to support research funding bodies and researchers to pursue research priorities. Such an approach should encourage priority setting partnerships to ensure the prioritised research uncertainties can be realistically answered by 
researchers within reasonable resource parameters. Non-specific research uncertainties, for example, questions which broadly ask the cause, treatment, or prognosis of a healthcare condition should be discouraged as they are not specific enough for research funders and researchers to assess appropriately.

Answering prioritised research uncertainties would represent a key step forward in improving clinical care. However, our specialty should acknowledge the important role of special interest groups in raising funding for the topic of their particular interest, research which stems from the intellectual curiosity of individuals, and fundamental research which does not have an immediate clinical application. A blended research strategy should off the optimal pathway to improving clinical care and patient outcomes.

\section{Conclusion}

Future research priority setting studies should publish a protocol, use formal consensus development methods capable of engaging with key stakeholders including women and their families, and ensure their methods and results are comprehensively reported. By developing prioritised list of research uncertainties, created to specifically highlight the most pressing clinical needs as perceived by communities, women, families, healthcare professionals, and others should help funding organisations and researchers to set their future priorities.

\section{Acknowledgements}

We would like to thank David J. Mills for administrative and material support.

\section{Conflicts of interest}

EC is supported by the National Institute for Health Research Manchester Biomedical Research Centre (IS-BRC-1215-20007). AH reports grants from the Chief Scientist Office Scotland, Ferring, Medical Research Council, National Institute for Health Research, Roche Diagnostics, and Wellbeing of Women, and consultancy fees from Abbvie, Ferring, and Roche. The remaining authors report no conflicts of interest. Completed disclosure of interest forms are available to view online as supporting information.

\section{Author contributions}

Study concept and design: LG, BI, LJG, and JMD. Acquisition of data: LG, BI, MS, LJG, and JMD. Analysis and interpretation of data: LG, BI, MS, EC, MP, LJG, AH, CMF, LAM, JS, and JMD. Drafting of the manuscript: JMD. Critical revision of the manuscript for important intellectual 
content: LG, BI, MS, MV, EC, MP, LJG, AH, CMF, JS, and LAM. Obtaining funding: JMD and CMF. Study supervision: JMD and CMF.

\section{Sources of funding}

This report is independent research supported by the Catalyst Fund, Royal Society of New Zealand. The Royal Society of New Zealand had no role in the study design, data collection and analysis, decision to publish, or preparation of the manuscript. EC is supported by the National Institute for Health Research Manchester Biomedical Research Centre (IS-BRC-1215-20007).

\section{Ethical approval}

Ethical approval not required.

\section{References}

1. Duffy J, Bhattacharya S, Herman M, Mol B, Vail A, Wilkinson J, et al. Reducing research waste in benign gynaecology and fertility research. BJOG: An International Journal of Obstetrics \& Gynaecology. 2017;124(3):366-9.

2. Chalmers I, Glasziou P. Avoidable waste in the production and reporting of research evidence. The Lancet. 2009;374(9683):86-9.

3. Tallon D, Chard J, Dieppe P. Relation between agendas of the research community and the research consumer. The Lancet. 2000;355(9220):2037-40.

4. Petit-Zeman S, Firkins L, Scadding JW. The James Lind Alliance: tackling research mismatches. The Lancet. 2010;376(9742):667-9.

5. James Lind Alliance. The James Lind Alliance Guidebook. Southampton, United Kingdom: National Institute for Health Research Evaluation, Trials and Studies Coordinating Centre; 2018. 6. Chien PF, Khan KS, Siassakos D. Registration of systematic reviews: PROSPERO. BJOG: An International Journal of Obstetrics \& Gynaecology. 2012;119(8):903-5.

7. Liberati A, Altman DG, Tetzlaff J, Mulrow C, Gøtzsche PC, loannidis JPA, et al. The PRISMA statement for reporting systematic reviews and meta-analyses of studies that evaluate healthcare interventions: explanation and elaboration. BMJ. 2009;339:b2700.

8. Horne AW, Saunders PTK, Abokhrais IM, Hogg L. Top ten endometriosis research priorities in the UK and Ireland. The Lancet. 2017;389(10085):2191-2.

9. RCOG World Congress 2019. Special Issue: Top Scoring Abstracts of the RCOG World Congress 2019, 17-19 June 2019, London, UK. BJOG: An International Journal of Obstetrics \& Gynaecology. 2019;126(S2):1-245. 
10. Prior M, Bagness C, Brewin J, Coomarasamy A, Easthope L, Hepworth-Jones B, et al. Priorities for research in miscarriage: a priority setting partnership between people affected by miscarriage and professionals following the James Lind Alliance methodology. BMJ Open. 2017;7(8):e016571.

11. Rees SE, Chadha R, Donovan LE, Guitard ALT, Koppula S, Laupacis A, et al. Engaging Patients and Clinicians in Establishing Research Priorities for Gestational Diabetes Mellitus. Canadian Journal of Diabetes. 2017;41(2):156-63.

12. Duley L, Uhm S, Oliver S. Top 15 UK research priorities for preterm birth. The Lancet. 2014;383(9934):2041-2.

13. Heazell AEP, Whitworth MK, Whitcombe J, Glover SW, Bevan C, Brewin J, et al. Research priorities for stillbirth: process overview and results from UK Stillbirth Priority Setting Partnership. Ultrasound in Obstetrics \& Gynecology. 2015;46(6):641-7.

14. Wan YL, Beverley-Stevenson R, Carlisle D, Clarke S, Edmondson RJ, Glover S, et al. Working together to shape the endometrial cancer research agenda: The top ten unanswered research questions. Gynecologic Oncology. 2016;143(2):287-93.

15. The Contraception Priority Setting Partnership. Collaboration, Choice, Care: The Contraception Priority Setting Partnership. London, United Kingdom: Faculty of Sexual and Reproductive Healthcare of the Royal College of Obstetricians and Gynaecologists; 2017. 16. HGPSP Steering Group. Hyperemesis Gravidarum Priority Settng Partnership Protocol. Southampton, United Kingdom: James Lind Alliance; 2018.

17. Pregnancy Hypertension Priority Setting Partnership. Protocol. Southampton, United Kingdom: James Lind Alliance; 2018.

18. Diabetes and Pregnancy Priority Setting Partnership. Protocol. Southampton, Untied Kingdom: James Lind Alliance; 2019.

19. Twins Research Australia. What research questions are important for the future health of twins and multiples? 2018 [cited 2019 03/07/2019]; Available from:

www.twins.org.au/research/current-studies/354-what-research-questions-are-important-for-thefuture-health-of-twins-and-multiples

20. Lam JR, Liu B, Bhate R, Fenwick N, Reed K, Duffy JMN, et al. Research priorities for the future health of multiples and their families: The Global Twins and Multiples Priority Setting Partnership. Ultrasound in Obstetrics \& Gynecology.Accepted for publication.

21. Priority Setting Partnership for Infertility. What should infertility research focus on next? London, United Kingdom: Priority Setting Partnership for Infertility; 2019. 
22. Contraception Prioity Setting Partnership. Contraception Top 10. 2019 [cited 07/07/2019]; Available from: www.jla.nihr.ac.uk/priority-setting-partnerships/contraception/top-10priorities.htm

23. Miscarriage Priority Setting Partnership. Miscarriage Top 10,.. 2018 [cited; Available from: www.jla.nihr.ac.uk/priority-setting-partnerships/miscarriage/top-10-priorities.htm

24. Saunders B, Sim J, Kingstone T, Baker S, Waterfield J, Bartlam B, et al. Saturation in qualitative research: exploring its conceptualization and operationalization. Quality \& Quantity. 2018 July 01;52(4):1893-907.

25. Murphy MK, Black NA, Lamping DL, McKee CM, Sanderson CF, Askham J, et al. Consensus development methods, and their use in clinical guideline development. Health technology assessment (Winchester, England). 1998;2(3):i-iv, 1-88. 
Table 1 Included research priority setting studies. * Developed in a single initiative, Priory Setting Partnership for Infertility.

\section{Setting}

\section{Protocol Method \\ yes/no}

\section{United Kingdom Yes}

International
James Lind Alliance method
James Lind Alliance method
Output

research uncertainties

10 research uncertainties
Peer reviewed

publication

yes/no

Endometriosis

Infertility

Male infertility *

Female and unexplained infertility *

Medially assisted reproduction *

Ethics, access, and organisation of infertility care *

\section{Miscarriage \\ Sexual and reproductive health}

United Kingdom Yes

\section{Contraception \\ Pregnancy and childbirth}

United Kingdom

Yes

James Lind Alliance method

10 research uncertainties

Yes

Gestational diabetes

Canada No

Preterm birth

Stillbirth

United Kingdom No

No

James Lind Alliance method

10 research uncertainties

No

\section{Gynaecological oncology}

Endometrial cancer

United Kingdom Yes

James Lind Alliance method

10 research uncertainties

James Lind Alliance method

15 research uncertainties

James Lind Alliance method

11 research uncertainties

Yes

UnitedKinadom Yes

James Lind Alliance method

10 research uncertainties

\section{Ongoing}

\section{Pregnancy and childbirth}

Twin and multiple pregnancies

Hyperemesis gravidarum

Blood pressure in pregnancy

Diabetes in pregnancy

$\begin{array}{ll}\text { International } & \text { Unclear } \\ \text { United Kingdom } & \text { Yes } \\ \text { United Kingdom } & \text { Yes } \\ \text { United Kingdom } & \text { Yes }\end{array}$

James Lind Alliance method

James Lind Alliance method

James Lind Alliance method

James Lind Alliance method 


\begin{tabular}{|ll|}
\hline Stage $\mathbf{1}$ & Gathering potential research uncertainties \\
\hline Systematic review & Research recommendations from Cochrane reviews and clinical practice guidelines \\
\hline Survey & Gathering research uncertainties from healthcare professionals, patients, and families \\
\hline Stage $\mathbf{2}$ & Verifying research uncertainties \\
\hline Systematic review & Confirming research uncertainties are unanswered \\
\hline Stage 3 & Interim priority setting \\
\hline Survey & Reducing the long list of research uncertainties to a short list \\
\hline Stage 4 & Final priority setting \\
\hline Consensus meeting & Prioritising research uncertainties and finalising the top ten \\
\hline
\end{tabular}

Figure 1 James Lind Alliance priority setting partnership method. 


\section{NIHR Health Technology Assessment, United Kingdom}

Priority Setting Partnership for Endometriosis. What are the outcomes and / or success rates for surgical or medical treatments that aim to cure or treat endometriosis, rather than manage it?

\section{NIHR Health Technology Assessment, United Kingdom}

Priority Setting Partnership for Infertility. In women with otherwise unexplained infertility does hysteroscopic removal of an endometrial polyp increase live birth rates?

NIHR Cochrane Incentive Award, United Kingdom

Priority Setting Partnership for Endometrial Cancer. Do changes in lifestyle, including weight loss, reduce the risk of recurrence and improve survival in women who have been treated for endometrial cancer?

\section{EVE Appeal, United Kingdom}

Priority Setting Partnership for Endometrial Cancer. Which women with abnormal vaginal bleeding should be referred for specialist review?

\section{Jon P. Moulton Charitable Foundation, United Kingdom}

Priority Setting Partnership for Endometrial Cancer. Which women with abnormal vaginal bleeding should be referred for specialist review?

Figure 2 Examples of research funders and researchers responding to research priority setting studies. 